\title{
PLANEJAMENTO EXPERIMENTAL NA DETERMINAÇÃO DO PONTO DE TURBIDEZ DOS TENSOATIVOS ULTRANEX
}

\author{
NEVES SILVA W. P. ${ }^{1}$, BARBOSA DE BARROS E. L. ${ }^{2}$, BARROS NETO E. L. ${ }^{1}$, CARVALHO \\ SANTOS J. $\mathrm{S}^{1}$., CARVALHO P. C. A. $\mathrm{P}^{1}$., LANDEIRA A. S. ${ }^{3}$ \\ ${ }^{1}$ Universidade Federal do Rio Grande do Norte, Programa de Pós Graduação em Engenharia Química \\ ${ }^{2}$ Universidade Federal do Rio Grande do Norte, Departamento de Engenharia Química \\ ${ }^{3}$ Universidade Federal do Rio Grande do Norte, Departamento de Engenharia de Alimentos \\ E-mail para contato: neves_wanessa@yahoo.com.br

\begin{abstract}
RESUMO - Alguns trabalhos encontrados na literatura comprovam a viabilidade do uso do ponto de turbidez de tensoativos não iônicos em eficientes métodos de extração para a separação, pré-concentração ou purificação de uma variedade de substâncias, incluindo íons metálicos e vários compostos orgânicos. A extração desses solutos se dá por uma característica particular dos tensoativos não iônicos, o chamado ponto de turbidez que tem origem nas propriedades que apresentam esses tensoativos. O objetivo deste trabalho é a determinação do ponto de turbidez dos tensoativos da linha ULTRANEX (nonilfenol etoxilados), com diferentes graus de etoxilação. O planejamento experimental possibilitou a determinação de um modelo significativo que prediz o ponto de turbidez do tensoativo em função dos graus de etoxilação e da concentração utilizada.
\end{abstract}

\section{INTRODUÇÃ̃o}

Os tensoativos são substâncias que pela sua estrutura e propriedades têm a capacidade de reduzir as tensões interfacial e superficial dos líquidos onde esses se encontram. Fazem parte da família de moléculas anfifílicas, que têm como característica duas regiões de solubilidade distintas e com distância suficiente para se comportarem de maneira independente, apesar de serem ligada uma a outra (DELNUNZLO, 1990); possuem em sua estrutura uma "cabeça" polar que é a parte hidrofílica e uma "cauda" apolar que é a parte hidrofóbica.

Os tensoativos não iônicos são caracterizados pelo ponto de névoa ou turbidez (HOLMBERG $e t$ al. 2004), que, pela própria constituição da molécula, a parte hidrofílica (óxido de eteno) diminui sua afinidade por água com o aumento da temperatura. Este fato faz com que o tensoativo, ao atingir a temperatura de turbidez em solução, promova uma separação de fases, sendo uma fase rica em tensoativo (coacervato) e outra apresentando baixa concentração do mesmo (fase diluída).

O fenômeno do ponto de turbidez com tensoativos não-iônicos tem sido utilizado em eficientes métodos de extração para a separação, pré-concentração ou purificação de uma variedade de 
substâncias, incluindo íons metálicos e vários compostos orgânicos. AL-GHAMDI et al. (2007), realizaram um estudo para avaliar o efeito do ponto de névoa de tensoativos não iônicos da família Triton-X (octilfenolpolietoxilado) em campos petrolíferos. SCHOTT (2002) realizou um estudo sobre o efeito de aditivos no ponto de turbidez em tensoativos não-iônicos, variando o seu grau de etoxilação. INOUE et al. (2003), determinaram a temperatura do ponto de turbidez de misturas de tensoativos não-iõnicos e fizeram uma modelagem termodinâmica.

Com base na importância citada, o presente trabalho fez uso do planejamento experimental para analisar o efeito da concentração e dos graus de etoxilação dos tensoativos da linha ULTRANEX no ponto de névoa dos mesmos.

Para descobrir e interpretar as influências da concentração e dos graus de etoxilação dos tensoativos da linha ULTRANEX sobre o ponto de névoa (ponto de turbidez) foi utilizado neste trabalho o planejamento experimental. Esta técnica é classificada como uma técnica do tipo simultânea, onde os fatores de interesse que realmente apresentam influências significativas na resposta são avaliados ao mesmo tempo. O planejamento fatorial foi executado e as variáveis independentes foram a concentração do tensoativo e o grau de etoxilação e a resposta analisada foi o ponto de névoa dos tensoativos. Os experimentos foram realizados em três níveis, máximo, mínimo e intermediário $(+1,-1$ e 0$)$.

\section{METODOLOGIA}

\subsection{Experimental}

A metodologia utilizada nos ensaios teve como base o procedimento descrito em LEMOS ARAÚJO (2010). As soluções foram preparadas com água destilada e deionizada e os reagentes utilizados foram de grau analítico. Todos os tensoativos analisados nesse trabalho pertencem à linha ULTRANEX (nonilfenóis polietoxilados). A fórmula geral dos tensoativos encontra-se representado pela Figura 01.

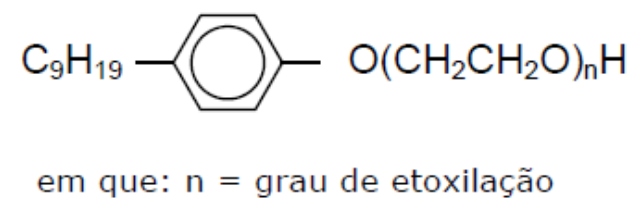

Figura 01 - Fórmula geral tensoativos ULTRANEX.

As propriedades dos tensoativos encontram-se dispostos na Tabela 01, onde o BHL representa o balanço hidrofílico/lipofílico dos tensoativos, que variam em função do número de unidades de óxido de eteno (graus de etoxilação). 
Tabela 01 - Propriedades dos tensoativos

\begin{tabular}{cccc}
\hline TENSOATIVO & $\begin{array}{c}\text { DESCRIÇÃO } \\
\text { QUÍMICA }\end{array}$ & $\begin{array}{c}\text { GRAUS DE } \\
\text { ETOXILAÇÃO }\end{array}$ & BHL \\
\hline ULTRANEX 80 & Nonilfenol 8 EO & 8 & 12,3 \\
ULTRANEX 95 & Nonilfenol 9,5 EO & 9,5 & 13 \\
ULTRANEX 110 & Nonilfenol 11 EO & 11 & 13,7 \\
\hline \multicolumn{4}{c}{ Fonte: Boletim Oxiteno }
\end{tabular}

Fonte: Boletim Oxiteno

As soluções foram preparadas num volume total de 100ml (água + tensoativo), conforme descrito no Planejamento Experimental. Após o preparo das soluções, as mesmas foram mantidas sob agitação constante no equipamento Water Separability Tester, a temperatura do banho foi aumentada gradativamente, até que a solução ficasse turva. Após a turvação, a temperatura da solução foi verificada por um termômetro digital e o valor considerado como ponto de névoa do tensoativo, na concentração específica.

\subsection{Planejamento Experimental}

O trabalho foi realizado em cinco etapas:

$1^{\text {o }}$ - Elaboração de modelos de regressão, com auxílio da ferramenta computacional STATISTICA 7.0, aplicando a técnica de Box-Behnken, utilizando alguns ensaios preliminares codificados conforme Tabela 02. Os valores de concentração de tensoativo variaram de 0,05 a 0,15 $\mathrm{g} / \mathrm{ml}$ e o grau de etoxilação variou de 8 a 11.

Tabela 02 - Codificação das Variáveis

\begin{tabular}{ccccc}
\hline & & \multicolumn{3}{c}{ NÍVEL CODIFICADO } \\
\cline { 3 - 5 } VARIÁVEIS INDEPENDENTES & SÍMBOLO & $\mathbf{- 1}$ & $\mathbf{0}$ & $\mathbf{1}$ \\
\hline Graus de Etoxilação & $\mathrm{EO}$ & 8 & 9,5 & 11 \\
Concentração de tensoativo $(\mathrm{g} / \mathrm{ml})$ & $\mathrm{Xt}$ & 0,05 & 0,1 & 0,15 \\
\hline
\end{tabular}

$2^{\circ}$ - Análise estatística do modelo obtido;

$3^{\circ}$ - Efeito das variáveis sobre o ponto de névoa dos tensoativos;

$4^{\circ}$ - Otimização dos modelos pela técnica de superfícies de resposta;

$5^{\circ}$ - Validação do modelo proposto. 


\section{RESULTADOS E DISCUSSÃO}

\subsection{Modelos de Regressão}

Os níveis dos fatores significativos e efeitos das interações entre os fatores que influenciam as respostas foram analisadas pelo planejamento fatorial $3^{2}$. Foram selecionados dois fatores importantes, conforme descrito na literatura: a concentração mássica de tensoativo (Xt) e o graus de etoxilação do tensoativo (EO), ambos em três níveis, planejados de acordo com a matriz codificada, descrita na Tabela 03. Esses fatores foram escolhidos em três níveis de $-1,0$ e 1 , valores baixo, médio e alto, respectivamente. A resposta foi o ponto de névoa (turbidez). Todos os ensaios experimentais foram realizados em duplicata e a média dos ensaios considerada como resposta final.

\begin{tabular}{cccc}
\multicolumn{4}{c}{ Tabela $03-$ Matriz do planejamento experimental } \\
\hline \multirow{3}{*}{ ENSAIO } & \multicolumn{2}{c}{ VARIÁVEIS } & RESPOSTA \\
& CONTROLADAS & \\
\cline { 2 - 4 } & EO & Xt (g/ml) & PN $\left({ }^{\circ} \mathbf{C}\right)$ \\
\hline 1 & -1 & -1 & 31,8 \\
2 & -1 & 0 & 36 \\
3 & -1 & 1 & 38,1 \\
4 & 0 & -1 & 52,1 \\
5 & 0 & 0 & 54,9 \\
6 & 0 & 1 & 59,0 \\
7 & 1 & -1 & 72,0 \\
8 & 1 & 0 & 72,8 \\
9 & 1 & 1 & 75,1 \\
\hline
\end{tabular}

A análise de regressão da resposta resultou em uma equação polinomial de segunda ordem, apresentada na Equação 01, que explica a relação do ponto de névoa do tensoativo ULTRANEX em função da concentração do tensoativo e do grau de etoxilação da molécula.

$P N=54,64+19,01 * E O+0,52 * E O^{2}+2,72 * X t-0,06 * X t^{2}-0,81 * E O * X t$

Onde PN é o ponto de névoa (turbidez) do tensoativo $\left({ }^{\circ} \mathrm{C}\right)$, EO é o número de unidade de óxido de eteno na molécula do tensoativo (graus de etoxilação) e Xt é a concentração de tensoativo na solução $(\mathrm{g} / \mathrm{ml})$.

\subsection{Análise Estatística do Modelo Obtido}

O modelo foi verificado pela Análise de Variância (ANOVA) apresentada na Tabela 04 e o coeficiente de determinação $\left(\mathrm{R}^{2}\right)$ foi usado como medida de correlação. Quanto maior a proximidade de $\mathrm{R}^{2}$ da unidade, melhor a correlação entre os valores preditos pelo modelo e os valores observados experimentalmente. 
Tabela 04- ANOVA

\begin{tabular}{lcccccc}
\hline FONTE DE & SQ & GL & MQ & $\mathbf{F}_{\text {cal }}$ & $\mathbf{F}_{\text {cal }} / \mathbf{F}_{\text {tab }}$ & SIGNIFICÂNCIA \\
VARIAÇÃo & & & & & & \\
\hline Regressão & 2215,004 & 5 & 443.008 & 476.344 & 52,866 & Modelo significativo \\
Resíduos & 2,978 & 3 & 0,93 & & & \\
\hline Total & 2217,982 & 8 & & & & \\
\hline
\end{tabular}

Coeficiente de Determinação $\mathrm{R}^{2}=0,996 ; \mathrm{F}_{5,3}=9,01$

$\mathrm{O}$ valor do teste $F$ calculado foi comparado ao valor do teste $F$ tabelado para a distribuição de $\mathrm{F}$ a 95\% de nível de confiança com os respectivos graus de liberdade. Como se pode observar na Tabela 04, o modelo apresentou regressão significativa em nível de $95 \%$ de confiança $\left(\mathrm{F}_{\text {calculado }}\right.$ superior ao $\mathrm{F}_{\text {tabelado }}$ ) com $\mathrm{R}^{2}=0,996$, evidenciando que o modelo explicou acima de $99 \%$ da variação dos dados experimentais.

\subsection{Efeito das variáveis sobre o Ponto de turbidez dos tensoativos}

Para avaliar o efeito da concentração do tensoativo e do grau de etoxilação da molécula no ponto de névoa (turbidez), o diagrama de Pareto foi analisado. No diagrama, obtido pelo software STATISTICA 7.0, as varáveis significativas no processo foram identificadas para um nível de confiança de 95\%. O Diagrama de Pareto é apresentado na Figura 02.

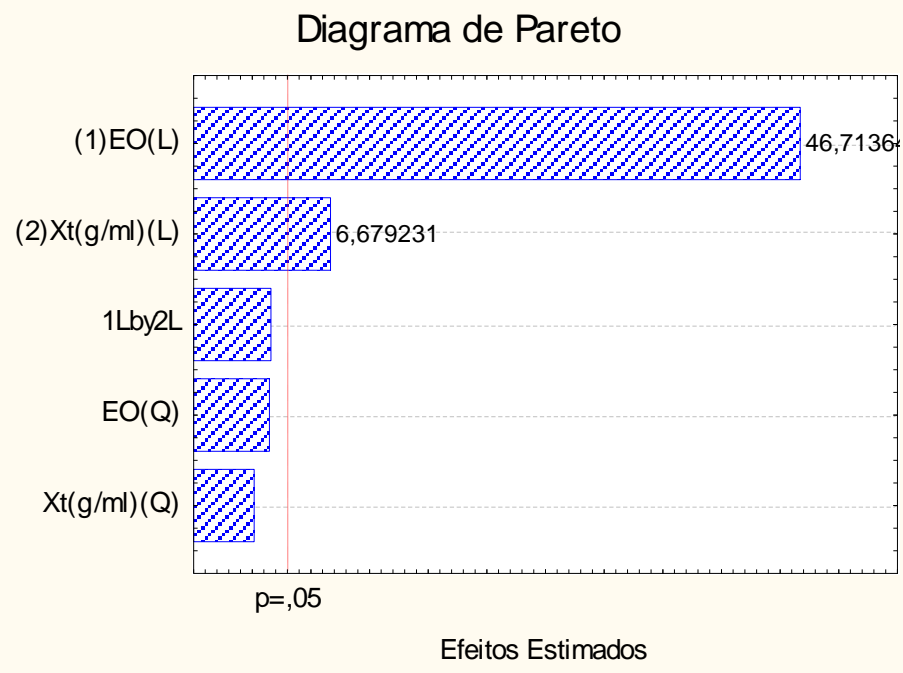

Figura 02 - Diagrama de Pareto

Observa-se pelo diagrama de Pareto que o grau de etoxilação da molécula e a concentração do tensoativo possuem efeito significaivo no ponto de turbidez. Verifica-se que ambas variáveis possuem efeito positivo no processo, resultando em uma relação diretamente proporcional das variáveis analisadas com o ponto de névoa, tal fato coincide com o comportamento verificado por SCHOTT (2002). Assim, se existe a necessidade de se trabalhar com pontos de névoa baixos, deve-se escolher 
tensoativos com grau de etoxilação baixo e concentração também baixa. O aumento dessas variáveis implicará em um aumento no ponto de névoa, resultando em custo operacional.

\subsection{Otimização do modelo pela técnica de superfície de Resposta}

Metodologia de superfície de resposta é uma ferramenta de estatística e métodos matemáticos que é útil para a modelagem e análise de problemas de engenharia. Nesta técnica, o objetivo principal é otimizar a superfície de resposta que é influenciado por vários parâmetros do processo, também quantifica a relação entre as variáveis independentes e as variáveis dependentes (respostas obtidas nas superfícies). A superfície de resposta gerada com os dados experimentais no programa STATISTICA 7,0 encontra-se disposta na Figura 03.

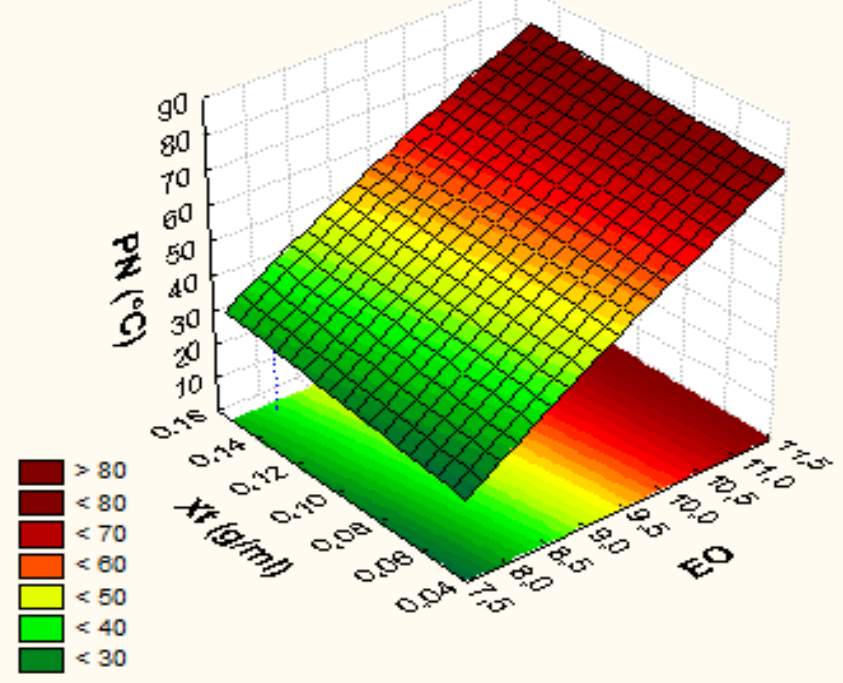

Figura 03 - Superfície de Resposta

Assim como observado no diagrama de Pareto, observa-se que o ponto de névoa é proporcional a concentração de tensoativo e ao número de óxido de eteno (graus de etoxilação) da molécula. Verifica-se que o grau de etoxilação é o fator com maior efeito no ponto de névoa, quanto maior a molécula, maior será o ponto de névoa. Esse fato pode ser explicado pela energia necessária para que o tensoativo diminua a afinidade pela água e, consequentemente alcance o ponto de névoa. Quanto maior a cadeia de óxido de eteno no tensoativo, maior a afinidade pela água e, maior a energia requerida para a diminuição de tal hidrofilia. Tal fato coincide com os resultados obtidos por SANTOS et al 2007, que concluiu que a cadeia do tensoativo influencia no ponto de turbidez.

Verifica-se que a região ótima (ponto de névoa mais baixo, inferior a $30^{\circ} \mathrm{C}$ ) ocorre nos tensoativos com grau de etoxilação igual a 8 e em concentração de abaixo de $0,06 \mathrm{~g} / \mathrm{ml}$.

\subsection{Validação do modelo obtido}


A Figura 04 ilustra a diferença entre os valores preditos pelo modelo e os valores reais (obtidos experimentalmente). Observa-se um comportamento válido do modelo, visto que o mesmo conseguiu prever os resultados experimentais com um desvio relativamente nulo.

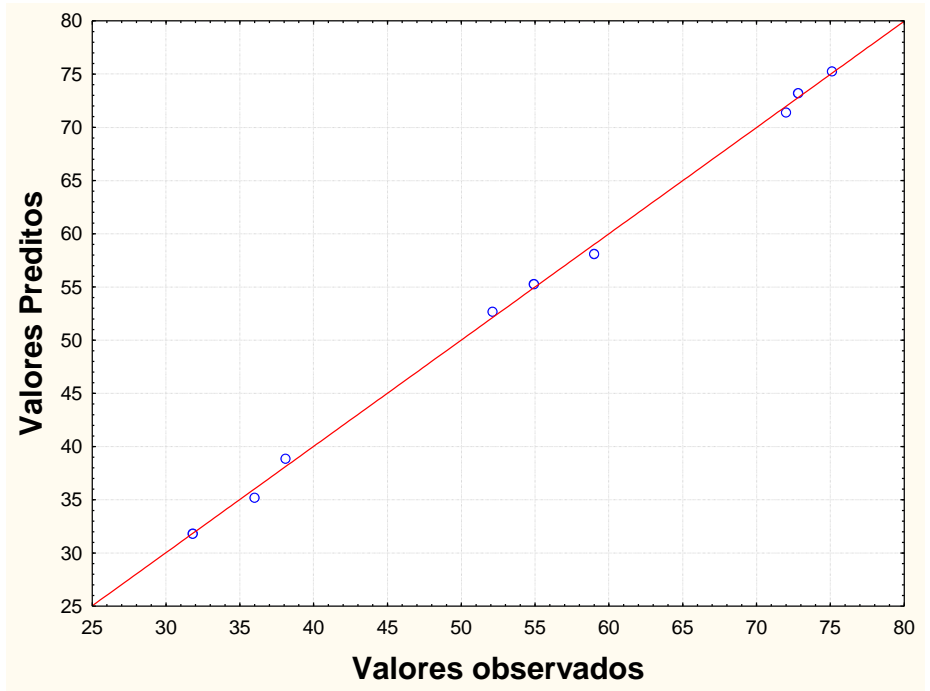

Figura 04 - Valores preditos e reais

Para verificar a eficiência do modelo proposto, os dados experimentais foram substituídos na Equação 01, e o ponto de névoa então calculado. Pode-se verificar pela Tabela 05, uma diferença máxima de $1,7^{\circ} \mathrm{C}$ entre o valor predito pelo modelo e o valor real, obtido experimentalmente. Vale salientar que uma diferença inferior a $2^{\circ} \mathrm{C}$ é considerada satisfatória em termos de temperatura de operação, e o modelo proposto torna-se válido para aplicação quando se deseja prever o ponto de turbidez do tensoativo estudado.

Tabela 05 - Ponto de névoa experimental e calculado

\begin{tabular}{|c|c|c|c|c|}
\hline \multicolumn{2}{|c|}{$\begin{array}{l}\text { VARIÁVEIS } \\
\text { CONTROLADAS }\end{array}$} & EXPERIMENTAL & MODELO & \multirow{2}{*}{$\begin{array}{l}\text { Diferença entre o } \\
\text { valor obtido e o } \\
\text { valor experimental }\end{array}$} \\
\hline EO & $\mathrm{Xt}(\mathrm{g} / \mathrm{ml})$ & PN $\left({ }^{\circ} \mathbf{C}\right)_{\text {real }}$ & PN $\left({ }^{\circ} \mathbf{C}\right)_{\text {calculado }}$ & \\
\hline-1 & -1 & 31,8 & 32,58 & 0,78 \\
\hline-1 & 0 & 36 & 36,16 & 0,16 \\
\hline-1 & 1 & 38,1 & 39,62 & 1,52 \\
\hline 0 & -1 & 52,1 & 51,86 & $-0,24$ \\
\hline 0 & 0 & 54,9 & 54,64 & $-0,26$ \\
\hline 0 & 1 & 59 & 57,30 & $-1,70$ \\
\hline 1 & -1 & 72 & 72,18 & 0,18 \\
\hline 1 & 0 & 72,8 & 74,16 & 1,36 \\
\hline 1 & 1 & 75,1 & 76,02 & 0,92 \\
\hline
\end{tabular}

EO, Xt e PN representa o grau de etoxilação da molécula, concentração de tensoativo (g/ml) e o ponto de névoa (turbidez) dos tensoatios, respectivamente. 


\section{9 a 22 de outubro de 2014 \\ Florianópolis/SC}

\section{CONCLUSÕES}

Os resultados obtidos experimentalmente e através do modelo matemático desenvolvido revelam que o grau de etoxilação da molécula e a concentração de tensoativo são diretamente proporcionais ao ponto de turbidez. Esse fato pode ser explicado pela energia necessária para que o tensoativo diminua a afinidade pela água e, consequentemente alcance o ponto de turbidez. Quanto maior a cadeia de óxido de eteno no tensoativo, maior a afinidade pela água e, maior a energia requerida para a diminuição de tal hidrofilia.

O modelo matemático desenvolvido neste trabalho é considerado o produto final do projeto e se mostra como ferramenta prática na simulação do ponto de névoa dos tensoativos da linha ULTRANEX. Os resultados calculados pelo modelo matemático apresentaram um comportamento próximo do real, com uma diferença máxima de $1,7^{\circ} \mathrm{C}$ do valor obtido experimentalmente.

\section{AGRADECIMENTOS}

Os autores agradecem o apoio financeiro, em forma de bolsa de estudos, dada pela CAPES e Petrobras.

\section{REFERÊNCIAS}

- $\quad$ AI-GHAMDI, A. M.; NASR-EL-DIN, H.; Effect of oilfield chemicals on the cloud point of nonionic. Colloid and Surface A, Arábia Saudita, n., p.5-18, 1997.

- DELnUNZLO, M. J. Tensoativos e suas aplicações básicas. Aerossol \& Cosméticos, 1422, 1990.

- LEMOS ARAÚJO, A. A.; Determinação experimental e modelagem termodinâmica do ponto de turbidez de sistemas aquosos com tensoativos nonilfenolpolietoxilados; Dissertação de mestrado, Universidade Federal do Rio Grande do Norte, Natal/RN, 2010.

- HOLMBERG, K.; JONSSON, B.; KRONBERG, B.; LINDMAN, B.; Surfactants and polymers in aqueous solution, $2^{\circ}$ Edição, Wiley, Inglaterra, 2004.

- INOUE, T,; OHMURA, H.; MURATA, D.; Cloud point temperature of polyoxyethylenetype nonionic. Colloid and Interface Science, Fukuoka-Japão, v.1, p,374-382, 2003.

- $\quad$ SANTOS, F. K. G.; ALVES, J. V. A.; CASTRO, DANTAS T. N.; DANTAS NETO, A. A.; DUTRA JR, T. V.; BARROS NETO, E. L.; Seleção de tensoativos não iônicos para uso na recuperação avançada de petróleo; $4^{\circ}$ PDPETRO, 2007.

- SCHOTT, H.; Effect of inorganic additives on solutions of nonionic. Colloid and Surface A, Filadelfia-EUA, v1., p.129-136, 2001. 